\title{
Spectrum of Angle Closure, Uveal Effusion Syndrome, and Nanophthalmos
}

\author{
${ }^{1}$ Eric Areiter, ${ }^{2}$ Matthew Neale, ${ }^{3}$ Sandra M Johnson
}

\begin{abstract}
Nanophthalmos, uveal effusion syndrome, and acute angle closure glaucoma (ACG) can present as a continuum in a patient, as is described here. This patient's angle closure was thought to be caused by idiopathic uveal effusion syndrome, and while there are no generally accepted diagnosis criteria for nanophthalmos, our patient fulfilled the criteria as defined by $\mathrm{Wu} .{ }^{10}$ To prevent development of further angle closure, the decision was made to do cataract extraction as opposed to medical management.
\end{abstract}

Keywords: Angle closure glaucoma, Cataract, Glaucoma, Nanonophthalmos, Uveal effusion syndrome.

How to cite this article: Areiter E, Neale M, Johnson SM. Spectrum of Angle Closure, Uveal Effusion Syndrome, and Nanophthalmos. J Curr Glaucoma Pract 2016;10(3):113-117.

\section{Source of support: Nil}

Conflict of interest: None

\section{INTRODUCTION}

Nanophthalmos is classically thought of as a rare condition characterized by a small eye in arrested development lacking many of the other malformations associated with eyes in the small-eye phenotype spectrum..$^{2-4}$ The etiology of nanophthalmos is derived from developmental arrest of the globe, usually affecting both eyes, after the embryonic fissure is closed. This results in a variety of associated clinical features, including an abnormally thickened sclera, short axial length, small corneal diameter, and crowding of the anterior chamber secondary to a high lens to eye volume ratio. ${ }^{5}$ Angle closure glaucoma (ACG) is also associated with nanophthalmos, due to progressive shallowing of the anterior chamber and angle narrowing with relative pupillary blocking. This most commonly will develop when a nanophthalmic patient is in his $30 \mathrm{~s}$ through 50 s. ${ }^{6}$ When considering nanophthalmos, other

\footnotetext{
${ }^{1,3}$ Resident, ${ }^{2}$ Ophthalmologist

${ }^{1}$ Department of Ophthalmology, Tulane Medical Center, New Orleans, Louisiana, USA

${ }^{2,3}$ Department of Ophthalmology, University of Virginia School of Medicine, Charlottesville, Virginia, USA

Corresponding Author: Eric Areiter, Resident, Department of Ophthalmology, Tulane Medical Center, New Orleans, Louisiana USA, Phone: +5049885263, e-mail: eareiter@tulane.edu
}

diagnoses, such as simple and posterior microphthalmos should also be examined. While nanophthalmos is associated with a small corneal diameter, posterior microphthalmos has a normal corneal diameter. ${ }^{7}$ Anterior microphthalmos has a horizontal corneal diameter of less than or equal to $11 \mathrm{~mm} .^{8}$

Nanophthalmic eyes are prone to problems related to their small biometry. ${ }^{1,6,9}$ No widely accepted biometric parameters for diagnosis exist; rather, the small-eye phenotype is best thought of as a clinical spectrum ranging from anophthalmos to axial hyperopia, and encompassing microphthalmos, nanophthalmos, segmental microphthalmos, and blepharophimosis. ${ }^{10}$

Angle closure glaucoma is a group of disorders that share a common pathway of mechanical obstruction of the trabecular meshwork. Traditional risk factors for ACG include demographic, genetic, anatomic, and environmental factors. ${ }^{11-14}$ Demographic factors include increased age and female gender, both of which correspond with a shallower anterior chamber depth. Certain ethnicities, such as Inuits and East Asians (particularly Chinese from Singapore and Hong Kong) are predisposed. ${ }^{14}$ Hyperopia is also associated with a decrease in anterior chamber angle and ACG.

Static biometric dimensions may not fully account for anatomic risk. ${ }^{5,15,16}$ Certain anatomic parameters of the eye are in constant flux; specifically, the volume of the uveal tract is subject to change. ${ }^{17}$ Another slower but progressive biometric parameter is lens size. Commonly, this volumetric change refers to an expansion of the choroid, resulting in anterior rotation of the ciliary body. Dynamic changes in choroidal thickness can be caused by changes in posture, ocular surgery, and the valsalva maneuver. ${ }^{18,19}$ For nanophthalmic patients, management of ACG initially consists of laser iridotomy, which is performed to relieve pupillary block. Argon-laser peripheral iridoplasty can then be performed if the anterior angle continues to remain closed following laser iridotomy. ${ }^{6}$ Iridoplasty can however induce a significant inflammatory response, causing choroidal effusions in the nanophthalmic eye. Systemic steroids, cycloplegics, and miotics can also be used with caution.

Idiopathic uveal effusion syndrome is thought to result from an abnormality in the sclera that impedes transscleral intraocular fluid outflow and compresses 
the vortex vein, leading to congestion of the choroidal veins. Intraocular fluid then accumulates in the choroid, leading to ciliochoroidal detachment. ${ }^{20}$

Three subtypes of idiopathic uveal effusion syndrome have been described by Uyama. Type I consists of a nanophthalmic eye with an average axial length of $16 \mathrm{~mm}$. Type II is a non-nanophthalmic eye with abnormal sclera and average axial length of $21 \mathrm{~mm}$ and presenting with a small refractive error. Types I and II are both described by Uyama as having disorganization of collagen fiber bundles and deposits of proteoglycans in the matrix. Type III is a non-nanophthalmic eye with normal sclera lacking collagen disorganization or deposits.

Gass and Jallow introduced subscleral sclerectomy in 1983. ${ }^{14}$ Uyama showed that the technique is effective in Types I and II for inducing resolution of serous subretinal fluid in these patients, while in type III this was not effective. Surgery on nanophthalmic eyes is infamous for complications and disappointment, however due to the lack of a generally accepted definition, patients should be evaluated individually for surgical eligibility. Ocular compression, choroidal effusions, pupillary block, and anterior chamber flattening must all be monitored for.

\section{CASE REPORT}

A 46-year-old Caucasian male presented with acute redness, watering, and painful visual loss in the right eye oculus dexter (OD). The community ophthalmologist measured an intraocular pressure (IOP) of $60 \mathrm{~mm} \mathrm{Hg}$ (Goldmann tonometer), and the patient was diagnosed with acute ACG. In addition to medical therapy, he was treated with laser peripheral iridotomy. Although IOP dropped into the normal range, the patient's anterior chamber remained very shallow. When corneal edema resolved, fundus examination revealed choroidal folds through the macula. He was referred to the Glaucoma Service at the University of Virginia with a diagnosis of nanophthalmos.

Examination findings of our initial consultation are summarized in Table 1 . Visual fields are shown in Figure 1.

The patient's angle closure was thought to be caused by idiopathic uveal effusion syndrome related to his short axial length and crowded anterior chamber. Although intraocular surgery in nanophthalmic eyes is relatively contraindicated, we felt that the risk of glaucoma recurrence was high enough to justify decompression of the anterior chamber by lens extraction. The patient received acetazolamide $500 \mathrm{mg}$ by mouth preoperatively. Prior to entering the eye, a large scleral flap was fashioned in the nasal quadrant, and was left unsutured at the conclusion of the case. The sclera
Table 1: Summary of ophthalmic examination

\begin{tabular}{|c|c|c|}
\hline & Oculus dexter & Oculus sinister \\
\hline $\begin{array}{l}\text { Va cc: }(\mathrm{W}:+5.25 \mathrm{sph} \\
+4.00+0.75 \times 150)\end{array}$ & $20 / 60$ & $20 / 20$ \\
\hline Manifest: & $20 / 30$ (+6.50 sph) & $\begin{array}{l}20 / 20-1 \\
(+4+0.75 \times 153)\end{array}$ \\
\hline Tonometry & $10 \mathrm{~mm} \mathrm{Hg}$ & $31 \mathrm{~mm} \mathrm{Hg}$ \\
\hline Pachymetry & 515 microns & 533 microns \\
\hline $\begin{array}{l}\text { Gonioscopy by } \\
\text { Sussman }\end{array}$ & TM inferiorly only & TM nasally only \\
\hline \multirow[t]{3}{*}{ Slit Lamp Biomicroscopy } & Very shallow AC & Shallow AC \\
\hline & $\begin{array}{l}\text { Corneal endothelial } \\
\text { pigment }\end{array}$ & $\mathrm{LPI} \times 1$ \\
\hline & $\mathrm{LPI} \times 2$ & \\
\hline Fundus & $\begin{array}{l}\text { Macular folds } \\
\text { present }\end{array}$ & Limited view \\
\hline $\begin{array}{l}\text { Optic Nerve Cup to Disk } \\
\text { Ratio }\end{array}$ & 0.1 & 0.1 \\
\hline Axial Length & $19.58 \mathrm{~mm}$ & $19.93 \mathrm{~mm}$ \\
\hline RNFL Thickness & 130 & 128 \\
\hline Anterior Chamber Depth & 2.31 & 2.14 \\
\hline Keratometry & $\begin{array}{l}46.87 \text { and } 48.35 \\
@ 88, \text { avg of } 47.61\end{array}$ & $\begin{array}{l}46.87 \text { and } 48.15 \\
@ 90 \text {, avg } 47.51\end{array}$ \\
\hline
\end{tabular}

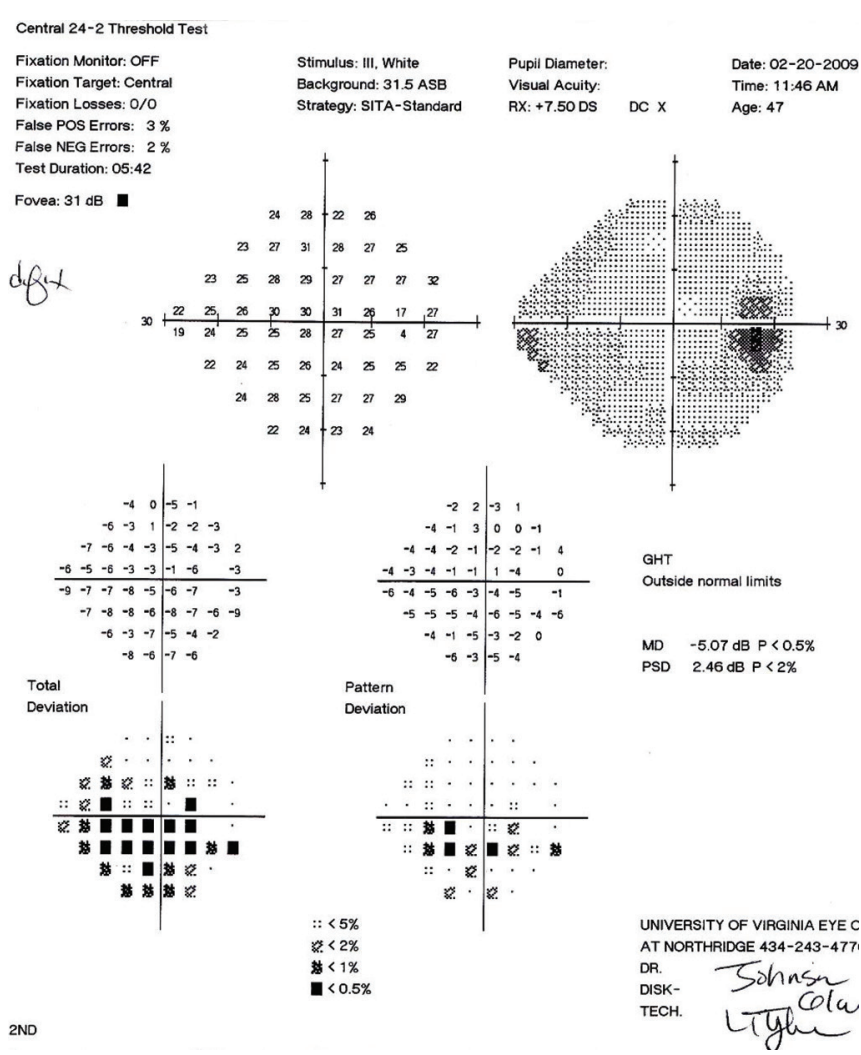

Fig. 1: Automated static perimetry

appeared to be of normal thickness; thus, a cut down to the uvea was not done.

Posterior synechiolysis, lensectomy, and a 34.0 D intraocular lens implantation were performed. Cycloplegia was maintained postoperatively. Postoperative day 1 , folds were again noted in the macula. They persisted, 


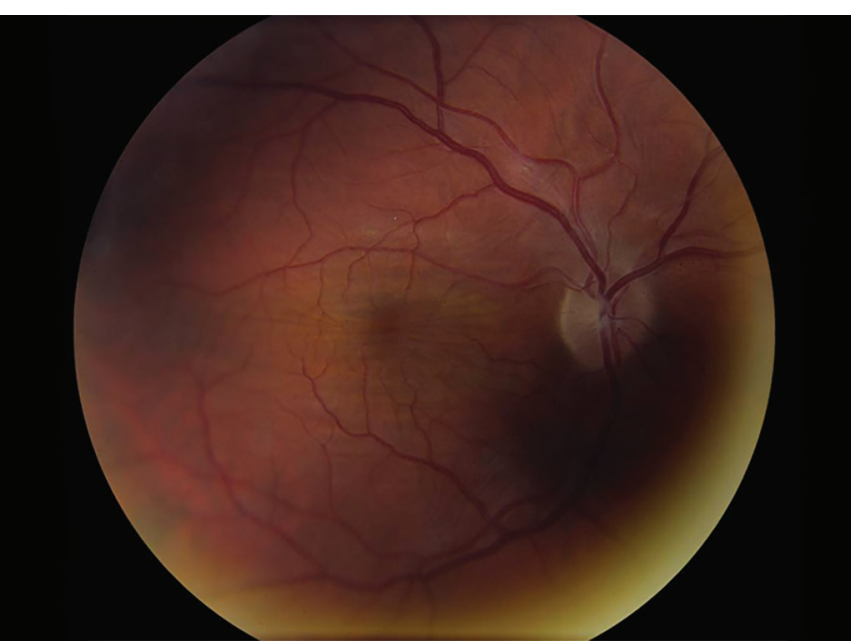

Fig. 2: Fundus photograph of macular folds

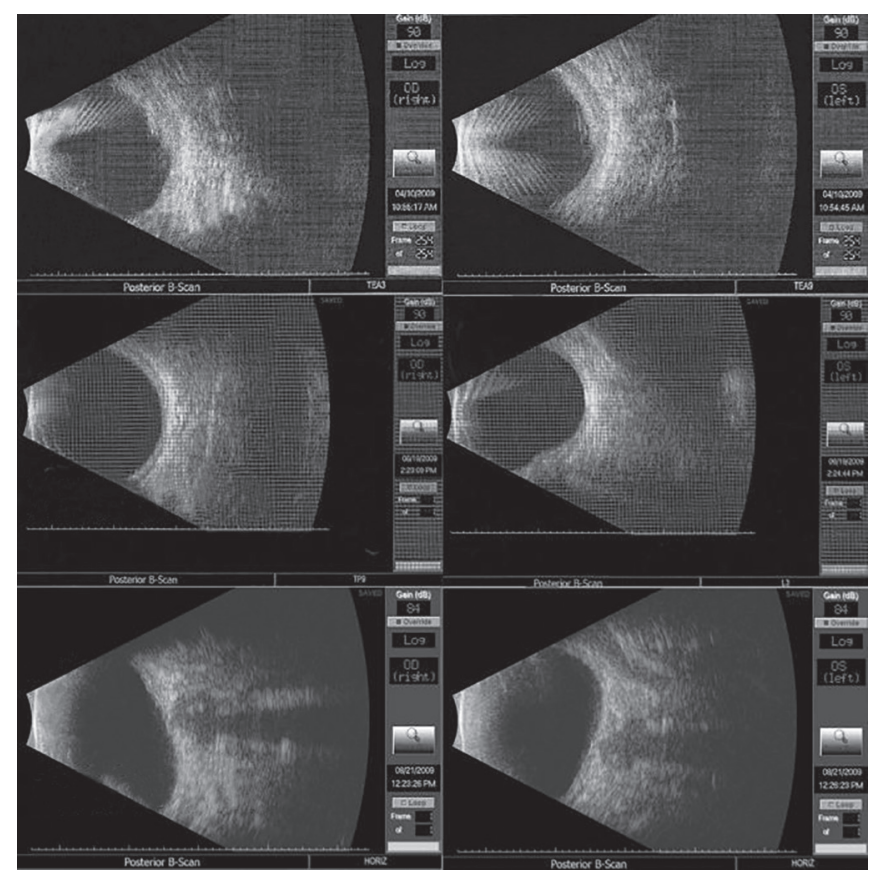

Fig. 3: Serial echography showing gradual diminution in choroidal thickness

and are demonstrated in the stereo fundus photograph seen in Figure 2.

With retinal consultation, a diagnosis of idiopathic uveal effusion syndrome was confirmed. Echography is shown at 2, 4 months, and at 6 months postoperatively in Figure 3.

Although not obtained preoperatively, Scheimpflug photography and anterior segment ultrasound taken 7 months postoperatively show open angles and suggest postoperative deepening of the anterior chamber in Figure 4 and anteriorly rotated ciliary processes in Figure 5. Left eye was found to be stable for the past several years and patient was status quo postperipheral iridectomy for narrow angle as well as maintained on medical therapy.

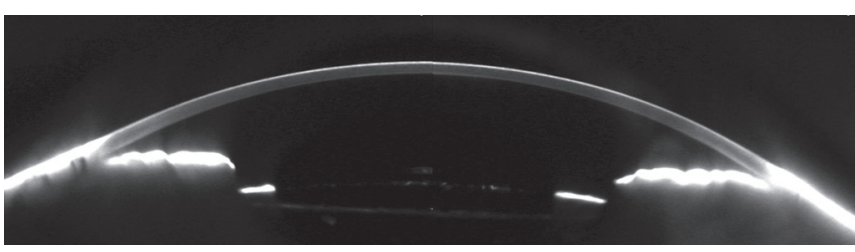

Fig. 4: Postoperative Sheimpflug photography of the right eye

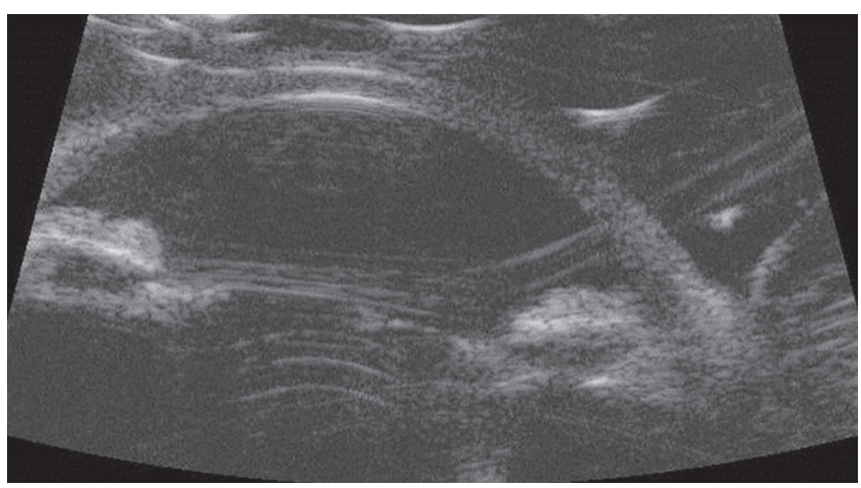

Fig. 5: Anterior segment ultrasound showing anteriorly rotated ciliary processes

\section{DISCUSSION}

The presence of an expanded choroid in a small eye is most consistent with a diagnosis of idiopathic uveal effusion syndrome. Uyama had relatively strict criteria for the diagnosis of nanophthalmos. Eyes had to have axial length less than $19.0 \mathrm{~mm}$, high hypermetropia, and thick, rigid sclera. The eye we report does not meet all of these criteria. Therefore, under Uyama's classification, this eye would be classified as type III (non-nanophthalmic eye with normal sclera). In Uyama's work, these eyes did not benefit from scleral surgery.

In other work, the definition of nanophthalmos has not been as firm. Wu considered eyes nanophthalmic if the anterior segment was crowded, if the eye was hyperopic, axial length was less than $21 \mathrm{~mm}$, and eye wall thickness in the posterior pole was greater than $1.4 \mathrm{~mm}$. Our patient meets these criteria. Similarly, Yalvac et $\mathrm{al}^{1}$ diagnosed nanophthalmos based on an axial length of less than $20.5 \mathrm{~mm}$, a shallow anterior chamber, high lens/eye volume ratio, moderate-to-severe hyperopia, and diffuse choroidal-scleral thickening as shown by echography.

The decision to perform cataract extraction was carefully made. Given that the primary defect was anatomic and not alleviated by laser iridotomy, and that an inferior arcuate scotoma was present, we decided that medical management was inadequate to prevent development of further angle closure. The more dense the lens and the more shallow the anterior chamber, the higher the risk of the corneal endothelium being jeopardized from the prior acute angle closure. ${ }^{21}$ Waiting for the development of elevated IOP would also contribute to the complexity of 
Table 2: Perioperative precautions for intraocular surgery on nanophthalmic eyes

\section{Preoperative}

- Control of elevated blood pressure and choroidal inflammation to decrease the risk of postoperative choroidal effusion

- Optimization of systemic blood pressure

- Subtenons dexamethasone $(1 \mathrm{~mL}$ of $10 \mathrm{mg} / \mathrm{mL})$

- Oral prednisone 60-120 mg daily, depending on weight

- Topical prednisolone acetate $1 \%$ every 2 hours while awake

- If both cataract and glaucoma surgery are indicated, risk may be minimized by performing a combined procedure.

- Intraocular lens (IOL) calculations should be done using SRK-T, Hoffer, and Holladay formulas, as they may be more accurate in highly hyperopic eyes.

- Silicone IOLs may be superior to acrylic as they are thinner. Intraoperative

- Intravenous mannitol at surgery to decrease vitreous pressure.

- Ocular compression.

- Creation of full-thickness anterior sclerotomies before entering the eye, and leaving them unsutured at the end of the case.

- A thick viscoelastic should be used to maximally protect the corneal endothelium.

- Phacoemulsification should be confined to the retropupillary and intrapupillary zones.

- Creation of a large peripheral iridectomy to ward off pupillary block.

- Goniosynechiolysis is controversial; one author cautions that the resulting increase in inflammation is not worth the potential benefit.

- Viscoelastic should be left in the eye at the conclusion of the case to avoid hypotony in combined procedures.

- Dexamethasone $10 \mathrm{mg}$ subtenons, and methylprednisolone $250 \mathrm{mg}$ IV at the conclusion of the case.

Postoperative

- Careful ophthalmoscopic exam for choroidal effusions; if present, admit the patient for

- serial IOP and BP monitoring

- high-dose IV steroids (methylprednisolone 250 mg IV every 6 hours)

- maintenance of IOP in mid-20s (using intracameral viscoelastic injections to raise the IOP if necessary).

the cataract extraction. Laser gonioplasty would have been technically challenging due to unfavorable iris curvature. Phacoemulsification in nanophthalmic eyes is relatively safe, though patients should be thoroughly examined for complications due to their increased likelihood., ${ }^{9,22}$

While this eye was, by nature of its small biometry, predisposed to angle closure, it is possible that the accumulation of the choroidal effusion was the precipitating factor in the patient's acute presentation. As described by Quigley, this relatively small change in the volume of the uveal tract may cause an already crowded eye to cross the threshold into Acute angle closure glaucoma (AACG) by a volumetric displacement of the lens-iris diaphragm. Choroidal volume increases are accompanied with pressure increases within the corneoscleral shell and lens forward movement. Quigley also believed that many eyes share the feature of nanophthalmos of having choroidal expansion that leads to forward lens movement even if they are not small enough to be considered nanophthalmic. ${ }^{19}$

When faced with the dilemma of a nanophthalmic eye in need of intraocular surgery, extensive precautions have been proposed to reduce the risk of choroidal effusion and unstable IOP. While we did not implement more than a few of those listed, Table 2 is a consolidated list of interventions that may improve the surgical risk-benefit quotient. Although they may be helpful, note that these are expert recommendations, and not evidence based. ${ }^{1,6,7,9}$

\section{CONCLUSION}

There seems to be a continuum between nanophthalmos, uveal effusion syndrome, and acute ACG as demonstrated by our patient. Overlapping nanophthalmos and uveal effusion syndrome in the absence of the effusions would present as a more straightforward primary angle closure patient.

\section{REFERENCES}

1. Yalvac IS, Satana B, Ozkan G, Eksioglu U, Duman S. Management of glaucoma in patients with nanophthalmos. Eye (Lond) 2008 Jun;22(6):838-843.

2. Singh OS, Simmons RJ, Brockhurst RJ, Trempe CL. Nanophthalmos: a perspective on identification and therapy. Ophthalmology 1982 Sep;89(9):1006-1012.

3. Brockhurst RJ. Nanophthalmos with uveal effusion. A new clinical entitiy. Arch Ophthalmol 1975 Dec;93(12):1989-1999.

4. Brockhurst RJ. Vortex vein decompression for nanophthalmic uveal effusion. Arch Ophthalmol 1980 Nov;98(1):1987-1990.

5. Liebmann JM, Weinreb RN, Ritch R. Angle-closure glaucoma associated with occult annular ciliary body detachment. Arch Ophthalmol 1998 Jun;116(6):731-735.

6. Burgoyne C, Tello C, Katz LJ. Nanophthalmia and chronic angle-closure glaucoma. J Glaucoma 2002 Dec;11(6):525-528.

7. Nouri-MahdaviK, Nilforushan N, Razeghinejad MR, AminiH, Perera SA. Glaucoma in a patient with nanophthalmos. J Ophthalmic Vis Res 2011 Jul;6(3):208-214.

8. Nihalani BR, Jani UD, Vasavada AR, Auffarth GU. Cataract surgery in relative anterior microphthalmos. Ophthalmology 2005 Aug;112(8):1360-1367.

9. Shields MB. Textbook of glaucoma. 4th ed. Baltimore (MD): Lippincott, Williams \& Wilkins; 1998.

10. Wu W, Dawson DG, Sugar A, Elner SG, Meyer KA, McKey JB, Moroi SE. Cataract surgery in patients with nanophthalmos: results and complications. J Cataract Refract Surg 2004 Mar;30(3):584-590.

11. See JLS, Chew PTK. Angle-closure glaucoma. In: Yanoff M, Duker J, editors. Ophthalmology. 3rd ed. St Louis (MO): Mosby Elsevier; 2009.

12. Ursin KV. On "spontaneous" choroidal detachment after glaucoma in the light of an exceptional case. Acta Ophthalmol (Copenh) 1965;43(6):751-760.

13. Pavlin CJ, Easterbrook M, Harasiewicz K, Foster FS. An ultrasound biomicroscopic analysis of angle-closure glaucoma secondary to ciliochoroidal effusion in IgA nephropathy. Am J Ophthalmol 1993 Sep 15;116(3):341-345. 
14. Amerasinghe N, Aung T. Angle-closure: risk factors, diagnosis and treatment. Prog Brain Res 2008;173:31-45.

15. Asai M, Tsujiguchi R, Taniguchi Y. Choroidal detachment developed in a case of acute closed angle glaucoma [in Japanese]. Jpn J Clin Ophthalmol 1988;42:1383-1386.

16. Sakai H, Morine-Shinjyo S, Shinzato M, Nakamura Y, Sakai $\mathrm{M}$, Sawaguchi S. Uveal effusion in primary angle-closure glaucoma. Ophthalmology 2005 Mar;112(3):413-419.

17. Quigley HA, Silver DM, Friedman DS, He M, Plyler RJ, Eberhart CG, Jampel HD, Ramulu P. Iris cross-sectional area decreases with pupil dilation and its dynamic behavior is a risk factor in angle closure. J Glaucoma 2009 Mar;18(3): 173-179.

18. Sihota R, Dada T, Aggarwal A, Srinivasan G, Gupta V, Chabra VK. Does an iridotomy provide protection against narrowing of the anterior chamber angle during Valsalva maneuvre in eyes with primary angle closure. Eye 2008;22(3):389-393.

19. Quigley HA, Friedman DS, Congdon NG. Possible mechanisms of primary angle-closure and malignant glaucoma. J Glaucoma 2003 Apr;12(2):167-180.

20. UyamaM,TakahashiK,KozakiJ,TagamiN, Takada Y,OhkumaH, Matsunaga H, Kimoto T, Nishimura T. Uveal effusion syndrome: clinical features, surgical treatment histologic examination of the sclera, and pathophysiology. Ophthalmology 2000 Mar;107(3):441-449.

21. Chen MJ, Liu CJ, Cheng CY, Lee SM. Corneal status in primary angle-closure glaucoma with a history of acute attack. J Glaucoma 2012 Jan;21(1):12-16.

22. Yuzbasioglu E, Artunay O, Agachan A, Bilen H. Phacoemulsification in patients with nanophthalmos. Can J Ophthalmol 2009 Oct;44(5):534-539. 
